ANNALES

POLONICI MATHEMATICI

$95.2(2009)$

\title{
Separation of global semianalytic sets
}

\author{
by Hamedou Diakite (Bamako)
}

\begin{abstract}
Given global semianalytic sets $A$ and $B$, we define a minimal analytic set $N$ such that $\bar{A} \backslash N$ and $\bar{B} \backslash N$ can be separated by an analytic function. Our statement is very similar to the one proved by Bröcker for semialgebraic sets.
\end{abstract}

1. Introduction. A global semianalytic set in a global analytic set $Z \subset \Omega, \Omega$ an open subset of $\mathbb{R}^{n}$, is a semianalytic subset of $Z$ admitting a description

$$
S=\bigcup_{i=1}^{p}\left\{x \in Z \mid f_{i}(x)=0, g_{i 1}(x)>0, \ldots, g_{i k_{i}}(x)>0\right\},
$$

where all the functions involved are analytic functions on the open set $\Omega$.

Of course a global semianalytic set is semianalytic in the usual local sense. The converse is not true in general; an example can be found in [CA96].

In particular, a global analytic set (often called a C-analytic set) is the zero set of finitely many global analytic functions.

This class of sets may be considered as the class of definable sets with respect to the ring $\mathcal{O}(\Omega)$, somewhat like the class of semialgebraic sets with respect to the ring of polynomials. The analogy stops here because many properties of semialgebraic sets are not known for global semianalytic sets; for instance, it is not known whether the closure or the connected components of a global semianalytic set are still global, except in some particular cases (sets of small dimension or compact sets). This is because the so called Artin-Lang property, which relates the definable sets of a ring of functions to the constructible sets of the real spectrum of the ring, does not hold for $\mathcal{O}(\Omega)$, not even in dimension one $([\mathrm{AB} 90])$. A weaker form of the Artin-Lang property was proved in the case $\operatorname{dim}(\Omega) \leq 2$ or when the ambient space is compact (see [Cas94], [CA96], and [ABR96] for a complete survey of those results). 
More recently, some general results were proved in [ABS05]. In this short note we will use those results to find a criterion to separate global semianalytic sets.

We shall consider global semianalytic sets $A, B$ in a global analytic set $Z \subset \Omega$. The set $Z$ has a well defined larger coherent ideal sheaf $\mathcal{J}_{Z} \subset \mathcal{O}_{\Omega}$ and we denote by $\mathcal{O}_{Z}$ the quotient sheaf, which is a coherent sheaf of $\mathcal{O}_{\Omega}$-modules. By Cartan's Theorem B, any global section of this sheaf is the restriction of an analytic function on $\Omega$. So when we speak of global analytic functions we always mean analytic functions on $\Omega$.

We will define the nullspace $N$ of $A$ and $B$, which is roughly speaking a minimal global analytic set such that $\bar{A}$ and $\bar{B}$ can be generically separated in irreducible sets outside $N$ (see Section 3 for a precise definition). We prove the following statement.

Theorem. Let $Z \subset \Omega$ be a global analytic set and let $A, B \subset Z$ be global semianalytic sets. Assume the dimension of their nullspace $N$ to be smaller than $\operatorname{dim}(Z)$. Then there is a function $f \in \mathcal{O}(\Omega)$ such that $f(\bar{A} \backslash N)>0$ and $f(\bar{B} \backslash N)<0$.

A more precise statement is already known for $\operatorname{dim}(Z)=1$ or 2 (see [Rui84] and [BP04]). A similar result was proved by Bröcker [Brö91] for semialgebraic sets, in a different formulation.

Note that this is not a result about closed global semianalytic sets since closures are not known to be global as we noticed before.

2. Definitions and preliminary results. We begin by defining what we mean by separation.

Definition 2.1. Two sets $P, Q \subset Z$ are separable if there is a function $g \in \mathcal{O}(\Omega)$ such that $g(P)>0$ and $g(Q)<0$. They are generically separable if there is a proper global analytic subset $Z^{\prime} \subset Z$ with $\operatorname{dim}\left(Z^{\prime}\right)<\operatorname{dim}(Z)$ and a function $g \in \mathcal{O}(\Omega)$ such that $g\left(P \backslash Z^{\prime}\right)>0$ and $g\left(Q \backslash Z^{\prime}\right)<0$.

Note that if one of the two sets is empty then separation holds trivially. Also note that closed sets are separable if and only if they are disjoint. So any function generically separating $P, Q$ should vanish on $(\bar{P} \cap Q) \cup(\bar{Q} \cap P)$.

The next result proves that obstructions to separation are located at the boundaries of the two sets. It slightly improves Theorem 4.2 in [ABS05], where the sets are supposed to be closed. We include the proof for the convenience of the reader.

Proposition 2.2. Let $A, B$ be global semianalytic sets in the global analytic set $Z \subset \Omega$. Let $X$ be a global analytic set containing $\bar{A} \cap \bar{B}$ and such that $\operatorname{dim}(X)<\operatorname{dim}(Z)$. Assume there is an open neighbourhood $U$ of $X$ and 
a function $f \in \mathcal{O}(\Omega)$ such that

$$
f(\bar{A} \cap U \backslash X)>0, \quad f(\bar{B} \cap U \backslash X)<0 .
$$

Then $\bar{A} \backslash X$ and $\bar{B} \backslash X$ are separable.

Proof. We can assume that $f$ vanishes on $X$ by replacing it by $f g$ for some positive global equation $g$ of $X$. Hence

$$
\{f=0\} \cap(\bar{A} \cup \bar{B}) \cap U=X \cap(\bar{A} \cup \bar{B}) \cap U .
$$

Since $X$ and $\Omega \backslash U$ are closed in $\Omega$ and disjoint, we can find an analytic function $h^{\prime}$ separating them with $X \subset\left\{h^{\prime}>0\right\} \subset U$. Hence we can assume that $U$ is an open global semianalytic neighbourhood of $X$.

So $(A \cup B) \cap U$ is a global semianalytic set. Hence, by [ABS05, Theorem 2.5], we can find a positive equation $h$ of $X$ such that $h<|f|$ on $(A \cup B) \cap U \backslash X$. We can assume $h<1$ on $U$, by shrinking it.

Take a smaller neighbourhood $V$ of $X$ with $\bar{V} \subset U$. Since $\bar{A} \backslash V$ and $\bar{B} \backslash V$ are closed and disjoint, there is $g \in \mathcal{O}(\Omega)$ such that $g(\bar{A} \backslash V)>0$ and $g(\bar{B} \backslash V)<0$; moreover, we can assume $|g| \geq 1$ on $\overline{A \cup B} \backslash V$.

Consider the open covering $\{U, \Omega \backslash \bar{V}\}$ and take a subordinate partition of unity $\left\{\sigma_{1}, \sigma_{2}\right\}$ such that $\sigma_{1 \mid V}=1$; then the smooth function $\varphi=\sigma_{1} f+\sigma_{2} g$ on $\mathbb{R}^{n}$ has the following properties:

- $\varphi_{\mid V}=f$.

- $\varphi(\bar{A} \backslash X)>0$ and $\varphi(\bar{B} \backslash X)<0$.

- $|\varphi(x)|>0$ on $\overline{A \cup B} \backslash V$.

Consider the function $\eta(x)$ defined as $(\varphi(x)-f(x)) / h^{2}(x)$ for $x \notin X$ and 0 for $x \in X$; it is well defined and smooth because $\varphi(x)-f(x)$ is identically zero on $V$.

Now, we want to find an analytic approximation $R$ of $\eta$ such that $f+R h^{2}$ separates $\bar{A} \backslash X$ and $\bar{B} \backslash X$.

To do this, take an exhaustive sequence $\left\{K_{m}\right\}$ of compact sets such that

$$
K_{i} \subset \stackrel{\circ}{K}_{i+1}, \quad \bigcup K_{m}=\Omega .
$$

Define

$$
r_{m}=\inf _{\left(K_{m+1} \backslash K_{m}\right) \cap((\bar{A} \cup \bar{B}) \backslash V)}|\varphi(x)|, \quad s_{m}=\sup _{K_{m+1} \cap((\bar{A} \cup \bar{B}) \backslash V)} h^{2}(x) .
$$

Note that $r_{m}>0$ since $|\varphi(x)|>0$ on $\overline{A \cup B} \backslash V$ and the set where we take the infimum is compact. Also $s_{m}>0$ because $X=\{h=0\} \subset V$. Finally, choose

$$
0<\varepsilon_{m}<\min \left(r_{m} / s_{m}, 1\right) .
$$

Applying Whitney's approximation theorem we find $R \in \mathcal{O}(\Omega)$ such that

$$
|R(x)-\eta(x)|<\varepsilon_{m} \quad \text { on } K_{m+1} \backslash \stackrel{\circ}{K}_{m} .
$$


Claim. $f+R h^{2}$ separates $\bar{A} \backslash X$ and $\bar{B} \backslash X$.

Proof. Assume first $x \in(\bar{A} \cup \bar{B}) \backslash V$; then, on $K_{m+1} \backslash \stackrel{\circ}{K}_{m}$,

$$
\left|\varphi(x)-\left(f+R h^{2}\right)(x)\right|=h^{2}(x)|\eta(x)-R(x)|<s_{m} \varepsilon_{m}<r_{m},
$$

and this proves that $\left(f+R h^{2}\right)(x)$ has the same sign as $\varphi(x)$.

If $x \in V \backslash X$ and $x \in K_{m+1} \backslash \stackrel{\circ}{K}_{m}$ we have $\eta(x)=0$ and $|R(x)|<\varepsilon_{m}<1$ so that

$$
|f(x)|-\left|R(x) h^{2}(x)\right|>|f(x)|-h^{2}(x) \geq h(x)-h^{2}(x)>0
$$

since $|h|<1$ on $V$. Hence $f+R h^{2}$ has the same sign as $f$ on $V \backslash X$.

The next fact is Lemma 4.5 of [ABS05].

Proposition 2.3. Let $Z$ be a global analytic set in $\Omega$ and $\left\{Z_{\alpha}\right\}_{\alpha}$ be the (locally finite) family of its global irreducible components having the same dimension as $Z$. Let $P, Q$ be subsets of $Z$. Then $P$ and $Q$ are generically separable in $Z$ if and only if for every $\alpha, P \cap Z_{\alpha}$ and $Q \cap Z_{\alpha}$ are generically separable in $Z_{\alpha}$.

Proof. One direction is clear: if $f$ generically separates $P$ and $Q$, its restriction to $Z_{\alpha}$ generically separates $P \cap Z_{\alpha}$ and $Q \cap Z_{\alpha}$. Conversely, assume $f_{\alpha} \in \mathcal{O}(\Omega)$ generically separates $P \cap Z_{\alpha}$ and $Q \cap Z_{\alpha}$. Consider the global analytic set having the same components as $Z$ (including the ones of smaller dimension) except $Z_{\alpha}$. Take a positive equation $p_{\alpha}$ of this global space. Then $f_{\alpha} p_{\alpha}$ vanishes on all irreducible components of $Z$ except $Z_{\alpha}$ and still generically separates $P \cap Z_{\alpha}$ and $Q \cap Z_{\alpha}$. So

$$
\sum_{\alpha} f_{\alpha} p_{\alpha}
$$

is well defined on $Z$ and generically separates $P$ and $Q$ because $\left\{Z_{\alpha}\right\}$ is a locally finite family.

3. Separation. Before entering the proof of the Theorem of the Introduction, we need some preparation.

Definition 3.1. The Zariski closure of a semianalytic set $A \subset Z \subset \Omega$ is the smallest global analytic subset of $Z$ containing it. It will be denoted by $\bar{A}^{Z}$.

REMARK 3.2. The Zariski closure of a semianalytic set can be very big if the set is not global: see for instance the examples in [CA96].

We do not know whether the boundary of a global analytic set $A \subset Z$ is global or not, unless $Z$ is a manifold of dimension $\leq 3$. Nevertheless, $\overline{\partial A}^{Z}$ is a global analytic set contained in the zero set of the product of the (finitely many) functions defining $A$. If $Z$ is irreducible, this implies 
$\operatorname{dim}\left(\overline{\partial A}^{Z}\right)<\operatorname{dim}(Z)$, and so, in this case, generic separation for two global semianalytic sets is the same as generic separation for their closures.

We shall repeatedly use the following key lemma, which is Corollary 2.8 in [ABS05].

Lemma 3.3. Let $S$ be a global semianalytic set in $Z$, and $g, h \in \mathcal{O}(\Omega)$ be global analytic functions. Then there exists $\varepsilon \in \mathcal{O}(\Omega), \varepsilon \geq 0$, such that:

(i) $g+\varepsilon h$ has the same sign as $g$ on $\bar{S}$;

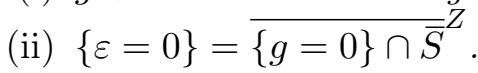

Next we define the nullspace of $A$ and $B$.

Definition 3.4. Let $A, B \subset Z$ be global semianalytic sets. We define the nullspace of $A$ and $B$ to be the union of all irreducible analytic subsets $Y$ of $Z$ such that $\bar{A} \cap Y$ and $\bar{B} \cap Y$ are not generically separable in $Y$.

Proposition 3.5. The nullspace $N$ of $A$ and $B$ is a global analytic set.

Proof. If $N=Z$, that is, $A$ and $B$ are not generically separable in any irreducible component $Z_{\alpha}$ of $Z$, there is nothing to prove. Otherwise, write $Z=Z^{\prime} \cup Z^{\prime \prime}$, where $Z^{\prime}$ consists of the irreducible components of $Z$ where $\bar{A} \cap Z_{\alpha}$ and $\bar{B} \cap Z_{\alpha}$ are not generically separable in $Z^{\prime}$, and $Z^{\prime \prime}$ consists of the others. Of course, $Z^{\prime} \subset N$. Now, by Proposition $2.3, \bar{A} \cap Z^{\prime \prime}$ and $\bar{B} \cap Z^{\prime \prime}$ are generically separable in $Z^{\prime \prime}$, i.e. there is a proper global analytic subset $W_{1}$ in $Z^{\prime \prime}$, with $\operatorname{dim}\left(W_{1}\right)<\operatorname{dim}\left(Z^{\prime \prime}\right)$, such that $\bar{A} \backslash Z^{\prime} \cup W_{1}$ and $\bar{B} \backslash Z^{\prime} \cup W_{1}$ are separable. Thus $Z^{\prime} \subset N \subset Z^{\prime} \cup W_{1}$. In fact, if $Y_{1}$ is an irreducible global analytic subset of $Z^{\prime \prime}$ and $Y_{1} \nsubseteq W_{1}$, then $\bar{A} \cap Y_{1}$ and $\bar{B} \cap Y_{1}$ are generically separable since $\bar{A} \cap Y_{1} \backslash W_{1} \cap Y_{1}$ and $\bar{B} \cap Y_{1} \backslash W_{1} \cap Y_{1}$ are separable and $\operatorname{dim}\left(W_{1} \cap Y_{1}\right)<\operatorname{dim}\left(Y_{1}\right)$.

Now, we can write $W_{1}=W_{1}^{\prime} \cup W_{1}^{\prime \prime}$, where $W_{1}^{\prime}$ consists of the irreducible components of $W_{1}$ where $\bar{A}$ and $\bar{B}$ are not generically separable, and $W_{1}^{\prime \prime}$ consists of the others. Then, of course, $W_{1}^{\prime} \subset N$. We find that $\bar{A} \cap W_{1}^{\prime \prime}$ and $\bar{B} \cap W_{1}^{\prime \prime}$ are also generically separable by Proposition 2.3 , so there is a proper global analytic subset $W_{2} \subset W_{1}^{\prime \prime}$, with $\operatorname{dim}\left(W_{2}\right)<\operatorname{dim}\left(W_{1}^{\prime \prime}\right)$, such that $\bar{A} \cap W_{1} \backslash Z^{\prime} \cup W_{1}^{\prime} \cup W_{2}$ and $\bar{B} \cap W_{1} \backslash Z^{\prime} \cup W_{1}^{\prime} \cup W_{2}$ are separable.

Thus $Z^{\prime} \cup W_{1}^{\prime} \subset N \subset Z^{\prime} \cup W_{1}^{\prime} \cup W_{2}$. In fact, if $Y_{2}$ is an irreducible global analytic subset of $Z^{\prime \prime}$ and $Y_{2} \nsubseteq W_{2}$, then $\bar{A} \cap Y_{2}$ and $\bar{B} \cap Y_{2}$ are generically separable since $\bar{A} \cap Y_{2} \backslash W_{2} \cap Y_{2}$ and $\bar{B} \cap Y_{2} \backslash W_{2} \cap Y_{2}$ are separable and $\operatorname{dim}\left(W_{2} \cap Y_{2}\right)<\operatorname{dim}\left(Y_{2}\right)$.

Hence, we can iterate this argument until we get a negative dimension of $W_{i}$. So there is $k \leq \operatorname{dim}(Z)$ such that

$$
Z^{\prime} \cup W_{1}^{\prime} \cup \cdots \cup W_{k}^{\prime} \subset N \subset Z^{\prime} \cup W_{1}^{\prime} \cup \cdots \cup W_{k}^{\prime} \cup W_{k+1},
$$

where $\operatorname{dim}\left(W_{k+1}\right)=-1$, hence $W_{k+1}=\emptyset$.

So $N=Z^{\prime} \cup W_{1}^{\prime} \cup \cdots \cup W_{k}^{\prime}$ is a global analytic set and we are done. 
Let us prove the Theorem of the Introduction.

Proof. Let $Z^{\prime}, Z^{\prime \prime}, W_{i}, W_{i}^{\prime}, W_{i}^{\prime \prime}$ for $i=1, \ldots, k$ and $W_{k+1}$ be the global analytic sets defined as in the proof of Proposition 3.5 such that $N=Z^{\prime} \cup$ $W_{1}^{\prime} \cup \cdots \cup W_{k}^{\prime}$, and $W_{k+1}=\emptyset$.

So $\bar{A} \backslash Z^{\prime} \cup W_{1}$ and $\bar{B} \backslash Z^{\prime} \cup W_{1}$ are separated, say, by a function $g_{1}$ in $\mathcal{O}(\Omega)$, and there is a function $h_{1}$ in $\mathcal{O}(\Omega)$ such that

$$
h_{1}\left(\bar{A} \cap W_{1}^{\prime \prime} \backslash W_{2}\right)>0, \quad h_{1}\left(\bar{B} \cap W_{1}^{\prime \prime} \backslash W_{2}\right)<0 .
$$

It is enough to prove the following recursive statement:

(*) Given $g_{i}, h_{i} \in \mathcal{O}(\Omega)$ such that

- $g_{i}\left(\bar{A} \backslash Z^{\prime} \cup W_{1}^{\prime} \cup \cdots \cup W_{i-1}^{\prime} \cup W_{i}\right)>0$, $g_{i}\left(\bar{B} \backslash Z^{\prime} \cup W_{1}^{\prime} \cup \cdots \cup W_{i-1}^{\prime} \cup W_{i}\right)<0$,

- $g_{i}\left(W_{i}\right) \equiv 0$ (if needed, multiply $g_{i}$ by a positive equation of $W_{i}$ ) and $h_{i} \in \mathcal{O}(\Omega)$,

- $h_{i}\left(\bar{A} \cap W_{i}^{\prime \prime} \backslash W_{i+1}\right)>0, h_{i}\left(\bar{B} \cap W_{i}^{\prime \prime} \backslash W_{i+1}\right)<0$,

there is a function $g_{i+1} \in \mathcal{O}(\Omega)$ such that

- $g_{i+1}\left(\bar{A} \backslash Z^{\prime} \cup W_{1}^{\prime} \cup \cdots \cup W_{i}^{\prime} \cup W_{i+1}\right)>0$, $g_{i+1}\left(\bar{B} \backslash Z^{\prime} \cup W_{1}^{\prime} \cup \cdots \cup W_{i}^{\prime} \cup W_{i+1}\right)<0$.

Indeed, it is enough to apply (*) successively to $Z \backslash Z^{\prime} \cup W_{1}, \ldots, Z \backslash Z^{\prime} \cup$ $W_{1}^{\prime} \cup \cdots \cup W_{i-1}^{\prime} \cup W_{i}, \ldots$, up to $Z \backslash N$. So, let us prove $(*)$.

Define

$$
\begin{aligned}
S= & \left(\left(A \backslash Z^{\prime} \cup W_{1}^{\prime} \cup \cdots \cup W_{i}^{\prime}\right) \cap\left\{h_{i} \leq 0\right\}\right) \\
& \cup\left(\left(B \backslash Z^{\prime} \cup W_{1}^{\prime} \cup \cdots \cup W_{i}^{\prime}\right) \cap\left\{h_{i} \geq 0\right\}\right) .
\end{aligned}
$$

Then $S$ is a global semianalytic set, and Lemma 3.3 yields $\varepsilon \in \mathcal{O}(\Omega), \varepsilon \geq 0$, such that

- $\operatorname{sign}\left(g_{i}+\varepsilon h_{i}\right)=\operatorname{sign}\left(g_{i}\right)$ on $\bar{S}$

- $\{\varepsilon=0\}={\overline{\left\{g_{i}=0\right\} \cap \bar{S}}}^{Z}$.

Now notice that

$$
\begin{aligned}
\left\{g_{i}=0\right\} & \cap \overline{A \cap\left\{h_{i} \leq 0\right\}} \subset\left\{g_{i}=0\right\} \cap \bar{A} \cap\left\{h_{i} \leq 0\right\} \\
& \subset\left(N \cup W_{i}\right) \cap \bar{A} \cap\left\{h_{i} \leq 0\right\} \subset N \cup\left(W_{i}^{\prime \prime} \cap \bar{A} \cap\left\{h_{i} \leq 0\right\}\right) \subset N \cup W_{i+1} .
\end{aligned}
$$

Since $N$ is global we get $\{\varepsilon=0\} \subset N \cup W_{i+1}$.

Put $g_{i+1}=g_{i}+\varepsilon h_{i}$. Then $g_{i+1}$ separates $\bar{A} \backslash Z^{\prime} \cup W_{1}^{\prime} \cup \cdots \cup W_{i-1}^{\prime} \cup W_{i}$ and $\bar{B} \backslash Z^{\prime} \cup W_{1}^{\prime} \cup \cdots \cup W_{i-1}^{\prime} \cup W_{i}$. Indeed, $x \in \bar{A} \backslash Z^{\prime} \cup W_{1}^{\prime} \cup \cdots \cup W_{i-1}^{\prime} \cup W_{i}$ implies $g_{i}(x)>0$ and if $x \in \bar{S}$, $\operatorname{sign}\left(g_{i+1}\right)=\operatorname{sign}\left(g_{i}\right)$; if $x \notin \bar{S}$, then $g_{i}(x)>0$ and $h_{i}(x) \geq 0$, hence $g_{i+1}(x)>0$. For $x \in \bar{B} \backslash Z^{\prime} \cup W_{1}^{\prime} \cup \cdots \cup W_{i-1}^{\prime} \cup W_{i}$, the same argument proves $g_{i+1}(x)<0$. 
Next we prove that $g_{i+1}$ separates outside $N \cup W_{i+1}$. In fact, if $\varepsilon(x) \neq 0$ and $x \in \bar{A} \backslash N \cup W_{i+1}$, then either $x \in \bar{A} \backslash Z^{\prime} \cup W_{1}^{\prime} \cup \cdots \cup W_{i-1}^{\prime} \cup W_{i}$ and so $g_{i+1}(x)>0$ as above, or $x \in \bar{A} \cap W_{i}$ and so $g_{i}(x)=0, h_{i}(x)>0$ and $\varepsilon(x)>0$. The same argument applies on $\bar{B} \backslash N \cup W_{i+1}$.

So $g_{i+1}$ actually separates $\bar{A} \backslash N \cup W_{i+1}$ and $\bar{B} \backslash N \cup W_{i+1}$, with $\operatorname{dim}\left(W_{i+1}\right)$ $<\operatorname{dim}\left(W_{i}\right)$. Hence $(*)$ is proved. Since $W_{k+1}=\emptyset, g_{k}$ separates $\bar{A} \backslash N$ and $\bar{B} \backslash N$, and we are done.

Remark 3.6. We can describe the nullspace $N$ of $A$ and $B$ as the zero set of two functions generically separating $\bar{A}$ and $\bar{B}$. Indeed, any function $g$ separating off $N$ can be easily modified to vanish on $N$, since $N$ is global analytic. Consider the constant function 1 and apply once more Lemma 3.3 to the set $S=A \cup B$. We find a function $\varepsilon \in \mathcal{O}(\Omega), \varepsilon \geq 0$, such that

- $\operatorname{sign}(g+\varepsilon)=\operatorname{sign}(g)$ on $\overline{A \cup B}$;

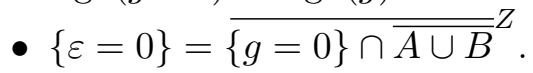

In particular, $g+\varepsilon$ generically separates $A$ and $B$. Of course, $N \subset\{g=0\}$. Also $N \supset\{g=0\} \cap \overline{A \cup B}$, hence $N$, being global analytic, contains the Zariski closure of that set, which is $\{\varepsilon=0\}$. Moreover, $g$ separates off $\{\varepsilon=$ $0\}$, hence $N \subset\{\varepsilon=0\}$.

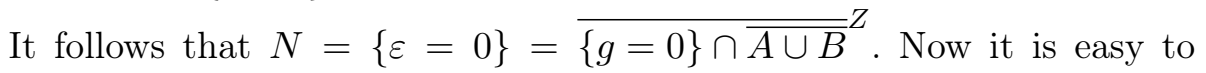
check that $N=\{x \in Z \mid g(x)=(g+\varepsilon)(x)=0\}$, since both vanish on $N$ but the second one does not vanish on the remaining part of the zero set of $g$.

\section{References}

[ABS05] F. Acquistapace, F. Broglia and M. Shiota, The finiteness property and Eojasiewicz inequality for global semianalytic sets, Adv. Geom. 5 (2005), 377-390.

[AB90] C. Andradas and E. Becker, A note on the real spectrum of analytic functions on an analytic manifold of dimension one, in: Real Analytic and Algebraic Geometry (Trento, 1988), Springer, Berlin, 1990, 1-21.

[ABR96] C. Andradas, L. Bröcker, and J. M. Ruiz, Constructible Sets in Real Geometry, Springer, Berlin, 1996.

[Brö91] L. Bröcker, On basic semialgebraic sets, Exposition. Math. 9 (1991), 289-334.

[BP04] F. Broglia and F. Pieroni, Separation of global semianalytic subsets of 2-dimensional analytic manifolds, Pacific J. Math. 214 (2004), 1-16.

[Cas94] A. Castilla, Artin-Lang property for analytic manifolds of dimension two, Math. Z. 217 (1994), 5-14.

[CA96] A. Castilla and C. Andradas, Connected components of global semianalytic subsets of 2-dimensional analytic manifolds, J. Reine Angew. Math. 475 (1996), 137-148. 
[Rui84] J. M. Ruiz, A note on a separation problem, Arch. Math. (Basel) 43 (1984), $422-426$.

DER de Mathématiques et Informatiques

Université de Bamako-FAST

BP: E3206, Bamako, Mali

E-mail: diakite@mail.dm.unipi.it

Received 21.5.2007

and in final form 25.7.2008 